\title{
The Issues of Designing Digital Platforms in the Paradigm of Open Systems
}

\author{
Olga Lukinova \\ Institute V.A. Trapeznikov Institute of Control Sciences of Russian Academy of Sciences \\ Moscow, Russia \\ lobars@mail.ru
}

\begin{abstract}
The methodological aspects of the design of digital economy platforms in terms of the open systems model are considered, and the interpretation of the interoperability stack for the use of it for integrating digital platforms into the ecosystem.
\end{abstract}

Keywords - digital economy, information system, OSE/RM model, digital platform, interoperability stack

\section{INTRODUCTION}

The current trend of the modern stage of the development of the world social system is the transition to a digital mode of existence, which is based on information and telecommunication technologies that penetrate all spheres of public life of individual states and entire continents. However, as emphasized in [1], at the moment there is no "concrete idea of the composition of the digital economy. In scientific articles, the authors consider the digital economy a priori as "everything that is done through digital technologies" and then analyze only its small components: the digital economy and the application of technology, retail trade and the digital economy, etc. [2] ". This limitation seems to be connected, on the one hand, with the multifactoriness of the concept of "digital economy", and on the other hand, with the absence of serious work in the field of the representation and development of digital economy objects.

In [3] it is emphasized that the process of digitalization should be considered in three aspects:

- Consumer, which forms new sociopsychological aspects of consumption, cultural and historical priorities for the development of the digital economy on the basis of a network of tightly interacting information systems.

- Management, which defines new principles, knowledge and technologies of management, designed to stabilize and improve the efficiency of the process and objectives of digitalization.

- Technological, accumulating new end-to-end technologies [4] to ensure the functioning of systems and their information exchange.

The article is devoted to understanding the implementation aspects of the technological layer as one of the system-forming structures of the digital economy.

\section{METHODOLOGICAL ASPECTS OF DESIGNING A DIGITAL ECONOMIC PLATFORMS}

In the acts and documents of various international, national and governmental organizations dealing with the problems of building of digital economy, such as the World Bank, the Organization for Economic Cooperation and Development (OECD), the BCS Institute (UK), the Eurasian Economic Commission (EEC), the Autonomous Non-Profit Organization «Digital Economy», Agency for Strategic Initiatives (Russia), etc. there appeared such terms as digital transformation, digital platform, end-to-end technologies, platform economy, cloud platform, information infrastructure, etc. These concepts formed the basis of the conceptual model of the technological (implementation) aspect of the digital economy (Fig. 1).

The current stage of development, as shown in the model, is characterized by digital transformation, which consists in changing the models of the activity of elements of the social system caused by the massive introduction of modern information and communication technologies. A public system is represented by the following subsystems [5]:

- Social, which determines the relationship between elements arising from their social status.

- Political, realizing relations for the purpose of ordering and safety of the vital elements of society.

- Moral, the purpose of which is relations related to the production and consumption by elements of the system of various intangible values.

- Economical, including relations that ensure the satisfaction of material needs of members of society. K. Marx considered this particular subsystem to be determinative, and the way of production of material values, which conditioned social, political and spiritual processes in society.

It should be emphasized that, in practice, there is a situation where the term "digital economy" in fact 
should be understood as a digital mode of activity in all 4 subsystems of public life.

It should be noted that the characteristic feature of the information society is the fact that the problem of determinism of a particular subsystem of social life is decided in favor of the prevalence of human interests. This means that the target value belongs precisely to one factor out of the three mentioned in the introduction [3] - consumer one. Organization of the implementation of objectives (management aspect) is carried out on the basis of business models, "working" in each of these public subsystems. Therefore, with digital transformation, the modification of business models should be carried out in accordance with the target criterion, reflecting the idea of obtaining an additional value (in comparison with the old business model) for the consumer from the introduction of information technology.

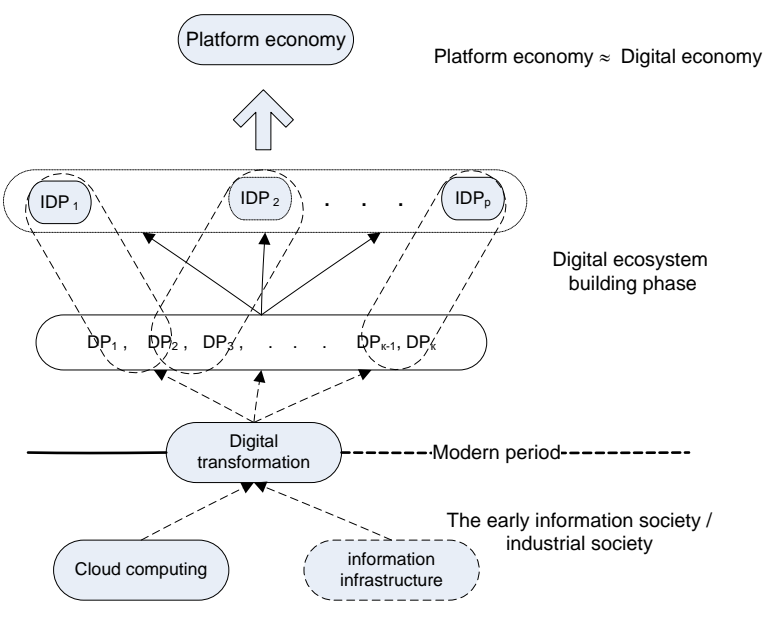

Fig. 1. Conceptual model of the technological layer of the digital economy

Thus, the beginning of the process of developing technological objects of the digital economy lies in the area of changing the business models of the society in accordance with the changing needs of the members of society, which are due to the widespread introduction of information technologies.

Technological prerequisites for the digital transformation of society were cloud technologies and the presentation of service-based information infrastructure, i.e. An extensible collection of standardized (or open-specification) information resources and services. At the same time, under the cloud service we mean a service that has the property of multitanancy - the sharing of providers and the sharing of users. In other words, public service is a standardized IT service that any provider can put on the market, and which can be used by any user $[6,9]$.

The basic technological objects supporting digital transformation are digital information platforms, which, according to [6], mean a combination of digital technologies, products or services that create the basis on which platform owners or external companies can create their own applications, technologies or services in various applied fields. It should be noted that in the Program "Digital Economy of the Russian Federation" [4] such application areas include:

- Platforms for research and development that organize networking between leading universities, research organizations, companies developing software for the implementation of digital economy technologies.

- Platforms for the management of fuel-energy, water, transport and other resources.

- Platform for drafting regulatory legal acts.

- A single centralized state cloud platform that provides storage and processing of all information generated by public authorities and local governments.

- An open public network platform for managing the results of intellectual activity.

- A platform for competence centers to be established on for each of the cross-cutting technologies directions, coordinating in the relevant subject areas all the research and training conducted in the country and responsible for the achievement of the world indicators for research and education.

- Pilot platforms for cooperating foreign and Russian organizations - partners of competence centers for technology transfer.

- Platforms that provide the services of makerspace for scientists on the basis of universities, scientific organizations, companies.

- National biometric platform for the creation of a national digital trust infrastructure.

- Platforms for "smart cities".

- Platforms for medical digital technologies.

The question is what is meant by the digital platform from the technological point of view and on what principles the design of the digital platform should be carried out.

Traditionally, the information system of any localization is accepted to be displayed in the open systems standards paradigm and to use the IEEE POSIX functional reference model OSE / RM (Open System Environment/Reference Model) [7, 8], which represents the IS in the form of two components:

- Applications (App), which are local (in the sense of the functional implementation of business functions of the "native" user) or shared cloud services.

- A platform that enables the functioning of applications through system services called by APIs. This component can provide its 
resources to external companies (users) in the modes: PAAS - to develop their own applications and further transfer them to external platforms, IAAS - for the functioning of external users' applications on this platform (external application). At the same time, the semantics of applications running on this platform, as a rule, does not depend on the subject domain, and the nature of system services does not reflect the specifics of this area. [9].

Detailed structuring of this model is presented in

In the next-generation information system, applications and platforms are changing their roles a bit. The differences are as follows.

Systems of a new generation, both digital platform, and applications that use this platform, are oriented to a specific subject area.

The platform component acquires an independent significance, i.e. a rigid connection between the platform and the applications of the information system is broken. Consumers or users of services (services) of the digital platform are usually not her "native" applications, but external ISs, other digital platforms, various mobile devices operating in the given domain (external service).

In the next generation of digital platforms, the OSE/RM platform as a whole remains the same: creating a unified environment for meeting application needs. But digital platform acquires application specificity. This means that part of the application's business logic is transferred to the digital platform level. Therefore in the literature there were such concepts as "industry-specific digital platform ", "application-specific digital platform ", etc. In this regard, the main tasks of the digital platform are as follows:

- Provide support for the subject matter of consumers. As already mentioned, the business logic, which provides a unified functional of a supporting character (for example, typical calculations, etc.), required for solving the problems of that domain, must be integrated into the platform, namely, the middleware system layer, which is devoted to the digital platform. Thus, the application users of the digital platform are freed from performing in some sense routine operations. Here, the problem arises of developing open API-interfaces oriented to specific subject areas. API is meant as a set of standard tools provided by some software object for the possibility of its use by an external program. As a rule, standard functions, classes, procedures are used in such a tools. In particular, in order for an application to be able to use the operating system's modules (services), there is a set of API functions called system calls. Each API must have a description of its signature and semantics. The signature of a means is the declaration (title) of a medium that allows one to identify it among others. For example, for WEBservice technology, the standard for describing service signatures is WSDL. Semantics is a description of the functionality of the medium in one form or another. A full description of the semantics of functions is the executable code of a function or class. Thus, there is an actual task of developing algorithms, code and signatures of APIinterfaces of the digital platforms.

- Ensure the functioning, mutual understanding and interaction of external users. It defines common rules and formats for data exchange, unified protocols and means for organizing interaction, common security requirements, publishes a list of services, etc. These service tasks can be performed by service applications or programs, also located at the middle ware level.

- Ensure effective administration of own resources in the interests of the owners of platforms. It can be implemented by local applications (the OSE / RM model administration plane [9]).

A higher level of generalization of applied business models for the implementation of complex solutions at the interface between application areas requires the interaction of various digital platforms and the creation of integrated digital platforms, i.e. we get a network of high-performance, intelligent, open to communication, dynamic IT systems that operate on the same principles and requirements. Such a construction forms a digital ecosystem, the totality of which, as shown in Fig. 1, is identical to the concept of the digital economy. The tasks of the ecosystem as a whole are the same as those of the digital platform, but on a larger scale: mutual understanding and alignment of versatile digital platforms, including low-level information systems, on the one hand, development of their subject, functional and interface components on the other.

To ensure the integration of the digital platform of any nature and from different suppliers within the ecosystem, it is advisable to refer to the stack of interoperability, the generalized form of which is based on the statement of the commission of the European Parliament [10] and is given below:

- Policy level - assumes that the information exchange between the digital platforms, users of the digital platform (applications) is carried out on the basis of agreed common goals;

- Normative level - involves interaction of the digital platform users in a single regulatory and legislative environment that implements common goals; 
- Organizational level - refers to the organizational aspects of the operation of platforms and their users and involves the integration of business models of various application areas in order to solve a complex problem;

- Semantic level - determines the ability of the digital platform and platform users to understand equally the meaning of the information they are exchanging;

- Syntactic level - determines the possibility of data exchange, the ability of platforms to integrate;

- Technical level - organization of the relationship between the digital platforms.

These principles of interoperability are valid not only for the integration of the digital platform, but, in a specific context, and for user interaction within the digital platform.

\section{CONCLUSION}

The article presents a conceptual model of technological concepts of digital economy, the possibility of using open standards for the model representation of digital platform and digital economy is shown. In addition, it is obvious that the implementation of all levels of the interoperability stack in the design of ecosystems will enable the creation of ecosystems that, in addition to the properties of self-organization, scalability and sustainability, have a specific feature: increasing the level of the ecosystem is improving the level and capabilities of each of the digital platforms that are in it.

In conclusion, I would like to emphasize that Russia, as the President of the Russian Federation underlines in various speeches, has also embarked on the path of accelerated digital development, the creation of ecosystems in all industries and spheres of life, with the subsequent expansion at the Eurasian Economic Union.

\section{REFERENCES}

[1] Yudina D., "Digital platform economy: definition and principles of functioning", http://uecs.ru/index.php?option=com flexicontent $\&$ view $=$ items\&id $=4582$

[2] Dobrynin, A. P.[and others], "Digital economy-various ways to effective application of technologies (BIM, PLM, CAD, IOT, Smart City, BIG DATA and others)", International Journal of Open Information Technologies, vol. 4. № 1, pp. 4$11,2016$.

[3] Tyurin V., "Nine problems that the ecosystem of digital platforms solves", https://www.itweek.ru/idea/article/detail.php?ID=196238

[4] Program " Digital economy of the Russian Federation», Order of the Government of the Russian Federation of 28 July 2017.

[5] http://www.grandars.ru/college/sociologiya/sferaobshchestva.html

[6] Boichenko A.V., Lukinova O. V. , "Platform for the digital economy", Proceedings of the 10th international conference "Electronic Kazan-2018", 2018.

[7] IEEE Std 1003.0-1005, IEEE Guide to the POSIX Open System Enviroment (OSE) - N-Y.: The Institute of Electrical and Electronics Engineers, 1995. - 194p.

[8] ISO/IEC TR 14252:1996. Information Technology. Guide to the POSIX Open System Environment (OSE).

[9] Lukinova O.V. "Methodological aspects of information system life cycle management based on functional standardization tools”, Software products and systems, № 4, pp. 27-35, 2016

[10] Communication from the Commission to the European Parliament, the Council, the European Economic and Social Committee and the Committee of Regions 'Towards interoperability for European public services, http://ec.europa.eu/isa/documents/isa_annex_ii_eif__en.pdf 